\section{Microbiological quality of kebabs sold in Palermo and Messina}

\section{Graziella Ziino, Giuseppe Gurrera, Chiara Beninati}

Dipartimento di Scienze Veterinarie, Università degli Studi di Messina, Messina, Italy

\section{Abstract}

Aim of this study was to evaluate the microbiological quality of kebabs retailed in Palermo and Messina. Twenty raw and 22 cooked kebab samples were analysed to determine the aerobic mesophilic bacteria (AMB), Enterobacteriaceae, Escherichia coli, sulphite reducing anaerobes, coagulase positive staphylococci, micrococci, Bacillus cereus and the presence of Salmonella spp. and Listeria monocytogenes. In raw kebabs, AMB ranged from 4.00 to $7.34 \mathrm{log}$ $\mathrm{cfu} / \mathrm{g}$ and Enterobacteriaceae from 1.00 to 7.59 $\log \mathrm{cfu} / \mathrm{g}$. Escherichia coli and sulphite reducing anaerobe counts were from $<1.00$ to 6.18 and $4 \log \mathrm{cfu} / \mathrm{g}$, respectively. Coagulase positive staphylococci ranged from $<1.00$ to 3.48 $\log \mathrm{cfu} / \mathrm{g}$ and micrococci from $<1.00$ to $6.00 \mathrm{log}$ $\mathrm{cfu} / \mathrm{g}$. Listeria spp. was found in three raw kebab samples. In cooked kebabs, the AMB values ranged from 1.78 to $6.30 \mathrm{log} \mathrm{cfu} / \mathrm{g}$, Enterobacteriaceae from 1.00 to $4.00 \mathrm{log} \mathrm{cfu} / \mathrm{g}$ and micrococci from $<1.00$ to $5.30 \mathrm{log} \mathrm{cfu} / \mathrm{g}$. Three samples were positive for Escherichia coli (from 1 to $1.30 \log \mathrm{cfu} / \mathrm{g}$ ) and one for sulphite reducing anaerobes (2.00 log cfu/g). Coagulase positive staphylococci were found in two samples with loads of 2.30 and 2.78 cfu/g, respectively. Salmonella spp., Listeria monocytogenes and Bacillus cereus were not isolated in raw and cooked samples. The results of this study show that the microbiological quality of kebabs sold in Palermo and Messina is quite variable. The authors stress the importance of selection of raw material of high quality as well as the control of cooking and storage temperatures in order to minimise bacterial potential hazards.

\section{Introduzione}

Il döner kebab è un piatto a base di carne, tipico della gastronomia turca, persiana e araba, divenuto popolare in America settentrionale e in Europa occidentale grazie all'immigrazione proveniente dal Medio Oriente. Successivamente si è diffuso un po' ovunque e oggi può trovarsi in numerose versioni, a seconda dei paesi e delle culture.

La carne (solitamente di bovino ma anche di pollo, tacchino, ovino e, solo nelle versioni occidentali, specie in Grecia, di maiale), tagliata a fettine e in parte triturata, viene condita 0 marinata, per almeno 3-6 h, con una grande varietà di spezie, secondo la richiesta, quali origano, menta, peperoncino, cannella, cumino, coriandolo, pepe, sale, olio, ecc. Successivamente essa viene impilata su uno spiedo metallico a formare un grosso cono, alternando le fettine con la parte trita e ponendo alla sommità parti grasse che, colando durante la cottura, evitano l'eccessivo essiccamento delle parti sottostanti. Una volta assemblato, il kebab può essere sottoposto a cottura, se consumato nel luogo di preparazione, o distribuito nei punti vendita. Più frequentemente, specie per i locali che lavorano in franchising, il kebab è di provenienza industriale, allo stato congelato, con pezzature variabili da 5 fino anche a $150 \mathrm{~kg}$.

Durante la cottura, oggi eseguita prevalentemente in sistemi dedicati a gas o elettrici, le temperature possono notevolmente oscillare in base alle dimensioni della preparazione e a seconda dello stato iniziale della materia prima. Infatti, specie nei kebab di grandi dimensioni, dopo qualche ora è possibile che all'esterno si raggiungano temperature anche di $85^{\circ} \mathrm{C}$ mentre all'interno permangano valori di $6-7^{\circ} \mathrm{C}$ (Cantoni, 2007). Il prodotto che non viene consumato interamente nell'arco della giornata è posto in cella frigorifero e riutilizzato in tempi successivi in base alla richiesta; così come può accadere che, in caso di ridotta affluenza dei clienti, l'operatore, per ridurre i consumi, spenga la fonte di calore con conseguente insufficiente cottura delle parti esterne (Cantoni, 2007).

Dopo la cottura il kebab è servito all'interno di panini (tradizionalmente pane arabo) 0 posto su un piatto vero e proprio con l'eventuale aggiunta di verdure miste e varie salse.

Il $\mathrm{pH}$ del prodotto crudo ha valori che oscillano tra 5.6 e 6.2. Per quanto concerne la composizione chimica si hanno le seguenti percentuali: acqua $55-60 \%$, grasso $20-28 \%$, proteine $15-17 \%$ e proteine non tissutali $12-14 \%$ (Cantoni, 2007).

Sotto il profilo igienico-sanitario, dai dati bibliografici, sembra emergere la possibilità che microrganismi potenzialmente patogeni per l'uomo, quali Salmonella spp., Staphylococcus aureus, Clostridium perfringens e, in minor misura, Escherichia coli 0157:H7 si possano isolare da questo alimento con una certa frequenza (Nichols et al., 1996; Bartholoma et al., 1997; Kasyisoglu et al., 2003; Vazgecer et al., 2004; Elmali et al., 2005; Ulukanli et al., 2006). Bryan et al. (1980), ad esempio, segnalano cariche di $10.000 \mathrm{ufc} / \mathrm{g}$ di $C$. perfringens subito sotto la superficie di Gyros o kebab commercializzati in Turchia. In
Correspondence: Graziella Ziino, Dipartimento di Scienze Veterinarie, Università degli Studi di Messina, viale Annunziata, 98168 Messina, Italy. Tel. +39.903503761 - Fax: +39.903503937 .

E-mail: gziino@unime.it

Key words: Kebab, Microbiological quality, Food hygiene, Sicily.

Received for publication: 15 January 2013.

Revision received: 26 March 2013.

Accepted for publication: 2 April 2013.

This work is licensed under a Creative Commons Attribution 3.0 License (by-nc 3.0).

(C) Copyright G. Ziino et al., 2013

Licensee PAGEPress, Italy

Italian Journal of Food Safety 2013; 2:e23

doi:10.4081/ijfs.2013.e23

una ricerca condotta da Jöckel e Stengel (1984) ben il 10\% del prodotto pronto al consumo conteneva $C$. perfringens. Percentuali del 19\% dello stesso microrganismo sono riportate da Stolle et al. (1993) in uno studio condotto su 44 campioni di kebab a Monaco, pur in presenza di cariche microbiche molto contenute. Krüger et al. (1993), su un totale di 40 campioni analizzati, hanno osservato la presenza di stafilococchi coagulasi positivi (coag. +) nel $42 \%$ dei casi con cariche di 5,3-6,2 log ufc/g. Che il kebab possa essere un alimento potenzialmente pericoloso per il consumatore è anche testimoniato dal fatto che sempre più frequentemente risulta essere l'alimento incriminato in episodi di malattia alimentare (Synnott et al., 1993; Evans et al., 1999).

Considerato che gli esercizi di somministrazione di döner kebab rappresentano una realtà sempre più diffusa nelle nostre città e che, sulla base di quanto ricordato in premessa, si tratta di un prodotto che ben si presta a potenziali problematiche d'ordine igienico-sanitario, abbiamo ritenuto interessante effettuare alcune valutazioni su questo alimento commercializzato nelle città di Palermo e Messina.

\section{Materiali e Metodi}

Lo studio è stato condotto, nel periodo compreso tra giugno e settembre del 2011, su 42 campioni di döner kebab, di cui 20 crudi e 22 cotti prelevati direttamente dai punti vendita, nelle città di Palermo (29 campioni) e Messina (13 campioni). A causa di motivi organizzativi o per mancanza di collaborazione da parte dei venditori, si riusciva ad ottenere porzioni crude e cotte dallo stesso cilindro solo per 15 campioni.

In un periodo compreso tra 1 e $3 \mathrm{~h}$, il kebab 
veniva trasportato, a temperatura di refrigerazione, in laboratorio. I campioni prelevati a Palermo erano composti prevalentemente da carne di pollo, tacchino e, più raramente, ovino. A Messina, invece, oltre a queste specie si reperivano kebab costituiti da carne suina (più precisamente si trattava di Gyros greco) $\mathrm{e}$ di agnellone. Nel 70\% dei casi la materia prima veniva acquistata congelata da stabilimenti industriali nazionali e, come riportato in etichetta, risultava additivata di stabilizzanti (E451, E450, E331, E500), antiossidanti (E300, E301), esaltatore di sapidità (E621), conservanti (E250) ma anche gelificanti, regolatori di acidità ecc. Oltre alle spezie erano presenti acqua, amido, proteine di soia o del latte, fibra e diversi zuccheri.

Il restante $30 \%$ dei campioni veniva acquistato direttamente presso macellerie locali. Il peso era compreso tra 2,5 e $20 \mathrm{~kg}$; solo un cilindro pesava $60 \mathrm{~kg}$. A detta dei proprietari dei punti vendita, una volta aperta la confezione 0 acquistato il prodotto fresco questo veniva consumato mediamente nell'arco di 1-2 giorni; il kebab invenduto veniva mantenuto in frigorifero a temperature comprese tra 0 e $4^{\circ} \mathrm{C}$.

I campioni crudi si presentavano nella totalità dei casi di aspetto e odore gradevole, le superfici carnee apparivano lucenti e riccamente speziate; in quelli cotti si accertava, invece, una certa variabilità del grado di cottura potendosi in alcuni casi apprezzare un colorito roseo delle porzioni meno superficiali.

Le analisi microbiologiche prevedevano la determinazione di: carica mesofila totale (CMT) in Plate Count Agar (Oxoid, Basingstoke, Hampshire, Gran Bretagna), incubato a $30^{\circ} \mathrm{C}$ per $72 \mathrm{~h}$; stafilococchi coag. + e micrococchi in Baird Parker Agar base (Oxoid) supplementato con Egg yolk tellurite emulsion (Oxoid), incubato a $37^{\circ} \mathrm{C}$ per $24 \mathrm{~h}$; Enterobacteriaceae in Violet Red Bile Glucose Agar (Oxoid), incubato a $37^{\circ} \mathrm{C}$ per $24 \mathrm{~h}$; Escherichia coli su Tryptone Bile X-glucuronide Medium (Oxoid), incubato a $44^{\circ} \mathrm{C}$ per $24 \mathrm{~h}$; anaerobi solfito riduttori in SPS agar (Oxoid), incubato a $37^{\circ} \mathrm{C}$ per $24 \mathrm{~h}$ in anaerobiosi; Bacillus cereus in Mannitol Egg Yolk
Polymixine Agar Base supplementato con polimixina e Egg yolk emulsion (Oxoid) a $30^{\circ} \mathrm{C}$ per $48 \mathrm{~h}$.

Si effettuava, inoltre, secondo metodiche interne, la ricerca di: i) Listeria monocytogenes, previo pre-arricchimento in Fraser Broth (0xoid) e successivo arricchimento in Buffered Listeria Enrichment Broth Base, entrambi incubati a $30^{\circ} \mathrm{C}$ per $21 \mathrm{~h}$; un'ansata delle brodo colture veniva infine strisciata su Listeria Selective Agar Base (Oxford) (Oxoid), incubato a $37^{\circ} \mathrm{C}$ per $24 \mathrm{~h}$; ii) Salmonella spp., con un pre-arricchimento in acqua peptonata tamponata incubata a $35^{\circ} \mathrm{C}$ per $18-20 \mathrm{~h}$; un successivo arricchimento in RappaportVassiliadis Enrichment Broth (0xoid) incubato a $41,5^{\circ} \mathrm{C}$ per $24-48 \mathrm{~h}$ e in Selenite Cystine Broth base (0xoid), incubato a $37^{\circ} \mathrm{C}$ per $18-24 \mathrm{~h}$; un'ansata delle brodocolture veniva strisciata in XLD (0xoid), incubato a $37^{\circ} \mathrm{C}$ per $24 \mathrm{~h}$.

Si prelevavano, quindi, tutte le colonie tipiche che, dopo ottenimento di patine singole, venivano sottoposte a test di valutazione delle caratteristiche morfologiche e biochimiche,

Tabella 1. Caratteristiche e risultati microbiologici di 30 campioni di kebab prelevati dallo stesso cilindro prima (15 campioni) e dopo (15 campioni) la cottura.

\begin{tabular}{|c|c|c|c|c|c|c|c|c|c|c|}
\hline $\begin{array}{l}\text { Tipologia } \\
\text { carne }\end{array}$ & $\begin{array}{c}\text { Materia } \\
\text { prima }\end{array}$ & $\begin{array}{l}\text { Peso } \\
\text { cilindro } \\
(\mathrm{kg})\end{array}$ & $\begin{array}{c}\text { Stato } \\
\text { campione }\end{array}$ & $\mathrm{pH}$ & $\begin{array}{l}\text { CMT } \\
(\log \\
\text { ufc/g) }\end{array}$ & $\begin{array}{c}\text { Enterobacteriaceae } \\
(\log \\
\mathrm{ufc} / \mathrm{g})\end{array}$ & $\begin{array}{l}\text { E. coli } \\
(\log \\
\mathrm{ufc} / \mathrm{g})\end{array}$ & $\begin{array}{l}\text { Anaerobi s.r. } \\
\begin{array}{c}(\log \\
\text { ufc/g) }\end{array}\end{array}$ & $\begin{array}{l}\text { Micrococchi } \\
(\log \\
\text { ufc/g) }\end{array}$ & $\begin{array}{c}\text { Stafilococchi } \\
\text { coag } \\
+(\log u f c / g)\end{array}$ \\
\hline Tacchino e vitello & $\mathrm{C}$ & 5 & $\begin{array}{l}\text { Crudo } \\
\text { Cotto }\end{array}$ & $\begin{array}{l}6.29 \\
6.20\end{array}$ & $\begin{array}{l}7,34 \\
2,60\end{array}$ & $\begin{array}{c}3,48 \\
<1,00\end{array}$ & $\begin{array}{l}2,00 \\
<1,00\end{array}$ & $\begin{array}{l}<1,00 \\
<1,00\end{array}$ & $\begin{array}{l}4,54 \\
2,60\end{array}$ & $\begin{array}{l}<1,00 \\
<1,00\end{array}$ \\
\hline Pollo e tacchino & C & 10 & $\begin{array}{l}\text { Crudo } \\
\text { Cotto }\end{array}$ & $\begin{array}{l}6.30 \\
6.27\end{array}$ & $\begin{array}{l}7,10 \\
2,40\end{array}$ & $\begin{array}{c}4,02 \\
<1,00\end{array}$ & $\begin{array}{c}2,00 \\
<1,00\end{array}$ & $\begin{array}{l}<1,00 \\
<1,00\end{array}$ & $\begin{array}{l}4,00 \\
2,30\end{array}$ & $\begin{array}{l}<1,00 \\
<1,00\end{array}$ \\
\hline Tacchino & $\mathrm{F}$ & 10 & $\begin{array}{l}\text { Crudo } \\
\text { Cotto }\end{array}$ & $\begin{array}{l}6.36 \\
6.26\end{array}$ & $\begin{array}{l}7,18 \\
1,78\end{array}$ & $\begin{array}{c}5,08 \\
<1,00\end{array}$ & $\begin{array}{c}4,34 \\
<1,00\end{array}$ & $\begin{array}{l}<1,00 \\
<1,00\end{array}$ & $\begin{array}{c}5,00 \\
<1,00\end{array}$ & $\begin{array}{l}<1,00 \\
<1,00\end{array}$ \\
\hline Tacchino e vitello & C & 5 & $\begin{array}{l}\text { Crudo } \\
\text { Cotto }\end{array}$ & $\begin{array}{l}6.69 \\
6.20\end{array}$ & $\begin{array}{l}6,95 \\
2,08\end{array}$ & $\begin{array}{c}3,17 \\
<1,00\end{array}$ & $\begin{array}{l}<1,00 \\
<1,00\end{array}$ & $\begin{array}{l}<1,00 \\
<1,00\end{array}$ & $\begin{array}{c}4,30 \\
<1,00\end{array}$ & $\begin{array}{c}2,00 \\
<1,00\end{array}$ \\
\hline Pollo e tacchino & $\mathrm{C}$ & 5 & $\begin{array}{l}\text { Crudo } \\
\text { Cotto }\end{array}$ & $\begin{array}{l}5.73 \\
5.71\end{array}$ & $\begin{array}{l}7,30 \\
6,30\end{array}$ & $\begin{array}{l}6,00 \\
4,00\end{array}$ & $\begin{array}{l}2,70 \\
1,00\end{array}$ & $\begin{array}{l}3,00 \\
2,00\end{array}$ & $\begin{array}{l}5,00 \\
5,00\end{array}$ & $\begin{array}{c}3,18 \\
<1,00\end{array}$ \\
\hline Pollo e tacchino & C & 5 & $\begin{array}{l}\text { Crudo } \\
\text { Cotto }\end{array}$ & $\begin{array}{l}5.85 \\
6.40\end{array}$ & $\begin{array}{l}7,30 \\
6,18 \\
\end{array}$ & $\begin{array}{l}6,30 \\
4,00\end{array}$ & $\begin{array}{l}2,85 \\
1,00 \\
\end{array}$ & $\begin{array}{l}<1,00 \\
<1,00\end{array}$ & $\begin{array}{l}6,00 \\
5,00\end{array}$ & $\begin{array}{l}3,48 \\
<1,00 \\
\end{array}$ \\
\hline Pollo e tacchino & $\mathrm{F}$ & 5 & $\begin{array}{l}\text { Crudo } \\
\text { Cotto }\end{array}$ & $\begin{array}{l}5.76 \\
5.80\end{array}$ & $\begin{array}{l}7,30 \\
4,70\end{array}$ & $\begin{array}{r} \\
6,00 \\
<1,00\end{array}$ & $\begin{array}{c}2,00 \\
<1,00\end{array}$ & $\begin{array}{l}<1,00 \\
<1,00\end{array}$ & $\begin{array}{l}5,30 \\
2,70\end{array}$ & $\begin{array}{c}3,43 \\
<1,00\end{array}$ \\
\hline Pollo e tacchino & $\mathrm{C}$ & 2,5 & $\begin{array}{l}\text { Crudo } \\
\text { Cotto }\end{array}$ & $\begin{array}{l}5.87 \\
5.98\end{array}$ & $\begin{array}{l}7,00 \\
4,30\end{array}$ & $\begin{array}{c}5,48 \\
<1,00\end{array}$ & $\begin{array}{l}<1,00 \\
<1,00\end{array}$ & $\begin{array}{c}3,00 \\
<1,00\end{array}$ & $\begin{array}{l}6,00 \\
2,70\end{array}$ & $\begin{array}{c}3,00 \\
<1,00\end{array}$ \\
\hline Pollo e tacchino & C & 10 & $\begin{array}{l}\text { Crudo } \\
\text { Cotto }\end{array}$ & $\begin{array}{l}5.75 \\
6.09\end{array}$ & $\begin{array}{l}7,18 \\
6,00\end{array}$ & $\begin{array}{l}6,00 \\
2,78\end{array}$ & $\begin{array}{c}2,48 \\
<1,00\end{array}$ & $\begin{array}{c}3,00 \\
<1,00\end{array}$ & $\begin{array}{l}5,48 \\
4,48\end{array}$ & $\begin{array}{l}3,30 \\
2,78\end{array}$ \\
\hline Pollo e tacchino & C & 5 & $\begin{array}{l}\text { Crudo } \\
\text { Cotto }\end{array}$ & $\begin{array}{l}5.85 \\
6.00\end{array}$ & $\begin{array}{l}7,18 \\
6,00\end{array}$ & $\begin{array}{l}5,70 \\
1,00\end{array}$ & $\begin{array}{c}1,70 \\
<1,00\end{array}$ & $\begin{array}{l}<1,00 \\
<1,00\end{array}$ & $\begin{array}{l}5,30 \\
4,90\end{array}$ & $\begin{array}{l}2,90 \\
2,30\end{array}$ \\
\hline Suino & $\mathrm{C}$ & 20 & $\begin{array}{l}\text { Crudo } \\
\text { Cotto }\end{array}$ & $\begin{array}{l}6.00 \\
6.20\end{array}$ & $\begin{array}{l}6,38 \\
3,54\end{array}$ & $\begin{array}{c}2,04 \\
<1,00\end{array}$ & $\begin{array}{l}<1,00 \\
<1,00\end{array}$ & $\begin{array}{l}<1,00 \\
<1,00\end{array}$ & $\begin{array}{c}3,46 \\
<1,00\end{array}$ & $\begin{array}{c}2,48 \\
<1,00\end{array}$ \\
\hline Agnellone & $\mathrm{C}$ & 10 & $\begin{array}{l}\text { Crudo } \\
\text { Cotto }\end{array}$ & $\begin{array}{l}6.10 \\
6.20\end{array}$ & $\begin{array}{l}6,77 \\
5,20\end{array}$ & $\begin{array}{c}3,72 \\
<1,00\end{array}$ & $\begin{array}{c}2,00 \\
<1,00\end{array}$ & $\begin{array}{c}1,00 \\
<1,00\end{array}$ & $\begin{array}{l}2,78 \\
4,48\end{array}$ & $\begin{array}{l}<1,00 \\
<1,00\end{array}$ \\
\hline Suino & $\mathrm{C}$ & 20 & $\begin{array}{l}\text { Crudo } \\
\text { Cotto }\end{array}$ & $\begin{array}{l}6.00 \\
6.22\end{array}$ & $\begin{array}{l}5,95 \\
4,76\end{array}$ & $\begin{array}{l}2,93 \\
<1,00\end{array}$ & $\begin{array}{l}<1,00 \\
<1,00\end{array}$ & $\begin{array}{l}<1,00 \\
<1,00\end{array}$ & $\begin{array}{l}3,65 \\
3,20\end{array}$ & $\begin{array}{l}<1,00 \\
<1,00\end{array}$ \\
\hline Agnellone & C & 10 & $\begin{array}{l}\text { Crudo } \\
\text { Cotto }\end{array}$ & $\begin{array}{l}6.05 \\
6.16\end{array}$ & $\begin{array}{l}5,43 \\
3,48 \\
\end{array}$ & $\begin{array}{c}1,00 \\
<1,00\end{array}$ & $\begin{array}{l}<1,00 \\
<1,00\end{array}$ & $\begin{array}{l}<1,00 \\
<1,00\end{array}$ & $\begin{array}{l}<1,00 \\
2,00\end{array}$ & $\begin{array}{l}<1,00 \\
<1,00\end{array}$ \\
\hline Suino & $\mathrm{C}$ & 20 & $\begin{array}{l}\text { Crudo } \\
\text { Cotto }\end{array}$ & $\begin{array}{l}5.96 \\
6.17\end{array}$ & $\begin{array}{l}6,23 \\
4,41\end{array}$ & $\begin{array}{l}3,11 \\
1,70\end{array}$ & $\begin{array}{c}2,34 \\
<1,00\end{array}$ & $\begin{array}{l}<1,00 \\
<1,00\end{array}$ & $\begin{array}{l}4,00 \\
3,04\end{array}$ & $\begin{array}{l}<1,00 \\
<1,00\end{array}$ \\
\hline
\end{tabular}

CMT, carica mesofila totale; E. coli, Escherichia coli; anaerobi s.r., anaerobi solfito riduttori; stafilococchi coag. + , stafilococchi coagulase positivi; C, congelata; F, fresca. 
quest'ultime mediante API 20 E e API Listeria (bioMérieux, Marcy l'Etoile, Francia).

Su tutti i campioni si eseguiva, infine, la misurazione del pH con pHmetro WTW pH330i.

\section{Risultati}

Le caratteristiche dei campioni analizzati, i risultati batteriologici e i valori di $\mathrm{pH}$ sono riportati nelle Tabelle 1 e 2 .

\section{Campioni crudi}

La CMT presentava valori compresi tra 4,00 e 7,34 log ufc/g. Sempre presenti le Enterobacteriaceae con valori tra 1,00 e 7,59 $\log \mathrm{ufc} / \mathrm{g}$, mentre Escherichia coli si isolava in 13 campioni con cariche comprese tra 1,70 e $6,18 \log$ ufc/g. Stafilococchi coag. + erano presenti in 10 campioni (tra 2,00 e 3,48 log ufc/g) mentre gli anaerobi solfito riduttori (s.r.), isolati in 6 campioni, presentavano cariche comprese tra 1,00 e 4,00 log ufc/g. I micrococchi si attestavano su valori tra 2,78 e 6,00 log ufc/g. In nessun caso si isolavano Salmonella spp., Listeria monocytogenes e Bacillus cereus; due campioni costituiti da carne di tacchino e vitello (congelati) e uno a base di tacchino (fresco) risultavano positivi per la presenza di Listeria spp.

\section{Campioni cotti}

Dopo trattamento termico, complessivamente si osservava una certa riduzione di tutti i parametri batteriologici con valori di CMT compresi tra 1,78 e $6,30 \mathrm{log}$ ufc/g. In tre campioni si isolava Escherichia coli (da 1,00 a 1,30 $\log \mathrm{ufc} / \mathrm{g}$ ) mentre in uno anaerobi s.r. (2,00 log ufc/g). Stafilococchi coag. + erano presenti in due campioni con valori rispettivamente di 2,30 e 2,78 log ufc/g; i micrococchi si attestavano su valori compresi tra 2,00 e 5,30 log ufc/g. Assenti Salmonella spp., Listeria monocytogenes, e Bacillus cereus.

Il pH nella materia prima oscillava tra 5.73 e 6.69 per i campioni congelati e tra 5.76 e 6.36 per quelli freschi. Dopo cottura, il pH si attestava su valori compresi tra 5.71 e 6.87 .

\section{Discussione}

I risultati da noi ottenuti sembrano dimostrare un'estrema variabilità microbiologica del kebab commercializzato nelle città di Palermo e Messina, in accordo con quanto già segnalato da diversi autori (Giaccone et al., 2006; Allegri, 2008; Nassi et al., 2010). Numerosi, infatti, sono i fattori che condizionano la microbiologia di questo alimento, quali le caratteristiche igieniche della materia prima, le tecniche di cottura, le successive fasi di stoccaggio, le condizioni igieniche del personale, nonché quelle dei locali e delle attrezzature.

Nell'indagine da noi condotta, complessivamente la materia prima, sia fresca che congelata, anche in presenza di conservanti, appariva di modesta qualità igienica, presentando in 11 campioni su 20 cariche batteriche di oltre 7 $\log$ ufc/g; ciò probabilmente anche in relazione all'impiego di carni avicole e alla grande quan- tità e varietà di aromi, così come frequente risultava la contaminazione ad opera di Enterobacteriaceae ed E. coli.

Il successivo trattamento termico, pur determinando una complessiva riduzione delle cariche microbiche, risultava in diversi casi non eseguito correttamente, come peraltro accertato all'esame organolettico. Al riguardo, ricordiamo, che sulla base delle linee guida elaborate dall'Advisory Committee on the Microbiological Safety of Food microbiological control of döner kebabs (2004), e in accordo con quanto proposto da Gilbert et al. (1996), volendo limitarci alla sola CMT, ben il 59\% dei nostri campioni sarebbe rientrato nella categoria non soddisfacente e il 14\% di essi, invece, in quella al limite dell'accettabilità. Nel kebab, dunque, considerata l'eventuale possibilità di abusi termici nei punti vendita o un prolungamento dei tempi di stoccaggio per una minore richiesta da parte del consumatore, così come peraltro descritto da altri autori (Cantoni, 2007), non è possibile escludere che si possano creare facilmente condizioni favorevoli alla crescita di microrganismi eventualmente presenti.

\section{Conclusioni}

Da quanto detto, fermo restando il mancato isolamento di germi patogeni nei campioni esaminati, emerge la necessità di operare la scelta di materie prime di qualità ineccepibile, nonché una corretta formazione del personale addetto alla preparazione e somministrazione

Tabella 1. Caratteristiche e risultati microbiologici di 5 campioni di kebab crudi e di 7 campioni cotti.

\begin{tabular}{|c|c|c|c|c|c|c|c|c|c|}
\hline $\begin{array}{l}\text { Tipologia } \\
\text { carne }\end{array}$ & $\begin{array}{c}\text { Materia } \\
\text { prima }\end{array}$ & $\begin{array}{l}\text { Peso } \\
\text { cilindro } \\
(\mathrm{kg})\end{array}$ & $\mathrm{pH}$ & $\begin{array}{l}\text { CMT } \\
(\log \\
\text { ufc/g) }\end{array}$ & $\begin{array}{c}\text { Enterobacteriaceae } \\
(\log \\
\mathrm{ufc} / \mathrm{g})\end{array}$ & $\begin{array}{l}\text { E. coli } \\
(\log \\
\mathrm{ufc} / \mathrm{g})\end{array}$ & $\begin{array}{l}\text { Anaerobi s.r. } \\
\text { (log } \\
\text { ufc/g) }\end{array}$ & $\begin{array}{c}\text { Micrococchi } \\
(\log \\
\text { ufc/g) }\end{array}$ & $\begin{array}{c}\text { Stafilococchi } \\
\text { coag } \\
+(\log u f c / g)\end{array}$ \\
\hline \multicolumn{10}{|l|}{ Campioni crudi } \\
\hline Tacchino e vitello & $\mathrm{C}$ & 5 & 6.17 & 6,24 & 3,59 & $<1,00$ & 3,00 & 4,07 & $<1,00$ \\
\hline Tacchino & $\mathrm{F}$ & 10 & 5.81 & 4,00 & 3,49 & $<1,00$ & $<1,00$ & 2,95 & $<1,00$ \\
\hline Tacchino & $\mathrm{F}$ & 10 & 6.30 & 6,96 & 7,59 & 6,18 & 4,00 & 4,85 & $<1,00$ \\
\hline Tacchino & $\mathrm{C}$ & 5 & 5.80 & 7,30 & 6,00 & 2,48 & $<1,00$ & 5,70 & 3,30 \\
\hline Agnellone & $\mathrm{F}$ & 10 & 5.83 & 7,30 & 6,30 & 4,78 & $<1,00$ & 4,78 & 3,48 \\
\hline \multicolumn{10}{|l|}{ Campioni cotti } \\
\hline Tacchino & $\mathrm{F}$ & 60 & 6.18 & 2 & $<1,00$ & $<1,00$ & $<1,00$ & 2,00 & $<1,00$ \\
\hline Tacchino & $\mathrm{F}$ & 5 & 6.27 & 4,36 & 1,60 & $<1,00$ & $<1,00$ & 2,60 & $<1,00$ \\
\hline Tacchino & $\mathrm{F}$ & 5 & 6.26 & 2,60 & $<1,00$ & $<1,00$ & $<1,00$ & 2,00 & $<1,00$ \\
\hline Pollo e tacchino & $\mathrm{C}$ & 5 & 6.25 & 6 & 3,30 & 1,30 & $<1,00$ & 5,00 & $<1,00$ \\
\hline Pollo e tacchino & $\mathrm{C}$ & 10 & 6.87 & 4,26 & $<1,00$ & $<1,00$ & $<1,00$ & 3,36 & $<1,00$ \\
\hline Pollo e tacchino & $\mathrm{C}$ & 10 & 6.81 & 4,04 & $<1,00$ & $<1,00$ & $<1,00$ & 2,48 & $<1,00$ \\
\hline Pollo e tacchino & $\mathrm{C}$ & 10 & 6.85 & 3,90 & $<1,00$ & $<1,00$ & $<1,00$ & 3,41 & $<1,00$ \\
\hline
\end{tabular}

CMT, carica mesofila totale; E. coli, Escherichia coli; anaerobi s.r., anaerobi solfito riduttori; stafilococchi coag. +, stafilococchi coagulase positivi; C, congelata; F, fresca. 
del kebab con particolare attenzione alla corretta applicazione delle temperature di cottura e di stoccaggio del prodotto.

\section{Bibliografia}

Allegri M, 2008. Aspetti igienico-sanitari e rischi per la produzione di kebab. Tesi di Laurea, Università di Padova, Italy. Disponibile al sito: http://85.46.58. 194/Tesi\%20Lauree/17-07-08/Tesi\%20 Allegri.pdf

Bartholoma A, Hildebrandt G, Stenzel WR, Erol I, 1997. A preliminary investigation of döner kebab in the German market. Fleischwirtschaft 77:913-5.

Bryan FL, Standley SR, HendersonWC, 1980. Time-temperature conditions of gyros. J Food Protect 43:346-53.

Cantoni C, 2007. Il kebab e il gyros-pita. Eurocarni 11:93-5.

Elmali M, Ulukanli Z, Tuzcu M, Yaman H, Cavli P, 2005. Microbiological quality of beef döner kebabs in Turkey. Arch Lebensmittelhyg 56:32-4.

Evans MR, Salmon RL, Nehaul L, Mably S,
Wafford L, Nolan-Farrell MZ, Gardner D, Ribeiro CD, 1999. An outbreak of Salmonella Typhimurium DT170 associated with kebab meat and yoghurt relish. Epidemiol Infect 122:377-83.

Giaccone V, Alberghini L, Catellani P, Milandri C, 2006. Caratteristiche microbiologiche del Kebab. In: Atti del XVI Convegno AIVI, 2006 giugno 22-24, Valenzano, Italy, p 329.

Gilbert RJ, de Louvois J, Donovan T, Hooper WL, Nichols G, Peel NR, Ribeiro CD, Roberts D, 1996. Microbiological guidelines for some ready-to-eat foods sampled at the point of sale. PHLS Microbiol Dig 13:41-3.

Jöckel S, Stengel G, 1984. [Döner Kebab Untersuchung und Beurteilung Einer Türkischen Spezialitat]. [Articolo in tedesco]. Fleiscwirthschaft 64:527-38.

Kasyisoglu S, Yilmaz I, Demirci M, Yetim H, 2003. Chemical and microbiological quality of the döner kebabs sold in Terkidag market. Food Control 14:469-74.

Krüger J, Schulz V, Kuntzer J, 1993. [Döner kebab-Unterschungen

zum Handelsbrauch in Stuttgart]. [Articolo in tedesco]. Fleischwirtschaft 73:1242-8.

Nassi R, Nuvoloni R, Forzale F, Pedonese F,
Gerardo B, Cambi L, D’Ascenzi C, 2010. Vendita di döner kebab nell'area lucchese: risultati dell'attività di sorveglianza. Rivista Italiana dell'AIVI 7:55-60.

Nichols G, Monsey H, de Louvois J, 1996. LACORS/PHLS study of the microbiological quality of döner kebab meat. Disponibile al sito: http://www.lacors.gov. uk/lacors/ home.aspx

Stolle A, Eisgruber H, Kerschhofer D, Krausse G, 1993. The gyros-study on variations of döner-kebab and microbiological and hygienic aspects in Munich. Fleisch wirtschaft 73:78-81.

Synnott M, Morse DL, Maguire H, Majid F, Plummer M, Leicester M, Threlfall EJ, Cowden J, 1993. An outbreak of Salmonella mikawasima associated with döner kebabs. Epidemiol Infect 111:473-81.

Ulukanli Z, Çavli P, Tuzcu M, 2006. Detection of Escherichia coli 0157:H7 from beef döner kebabs sold in Kars. Gazi University J. Science 19:99-104.

Vazgecer B, Ulu H, Oztan A, 2004. Microbiological and chemical qualities of chicken döner kebab retailed on the Turkish restaurants. Food Control 13:2614. 